\title{
Inhalt
}

Moralisieren im Alltag

Editorial

Paul S. Ruppel \& Anna Sieben

Haltung bewahren!

Ressentiment als verkörpertes,

implizit-moralisches Urteil

Markus Wrbouschek, Natalie Rodax,

Katharina Hametner, Sara Paloni

\& Nora Ruck

Strukturanalyse

normativ-praktischer Rationalität

Theoretische und methodologische Reflexionen

zur interpretativen Rekonstruktion

moralischer Orientierung

Ralph Sichler

Moralisierungen in Gastfamilien

für unbegleitete Geflüchtete

Differenzbearbeitung und Beziehungsgestaltung

zwischen Gast und Familie

Ines Gottschalk

Biografische Dynamiken

moralischer Positionierungen

in Prozessen sozialer Reintegration

Eine Längsschnittanalyse im Kontext

von Drogenkonsum, Kriminalität und Arbeit

Franz Zahradnik
3 "Egal was er auch ist,

Hauptsache einer von uns"

101

Eine sozial- und kulturpsychologische Analyse

zur Bedeutung von Moral im alevitisch-

12 sunnitischen Verletzungsverhältnis

Dilek A. Tepeli

"DA wurd ich Auch ganz blöde angeguckt» 124

Das moralische Spannungsfeld zwischen

gesellschaftlichem Anspruch und individueller

Integrität in einer Selbsthilfegruppe

zum Thema Übergewicht

34 Sarah Hitzler

Jenseits des guten Geschmacks?

Veganisierung im Alltag

Rebecca Thrun

Das klimaethische Selbst

56 als Subjektivierungsform

171

Eine idealtypische Konstruktion

Alexandria Krug

Impressum

191 


\section{鸪 Psychosozial-Verlag}

\section{Steven Taylor \\ Die Pandemie als psychologische Herausforderung Ansätze für ein psychosoziales Krisenmanagement}

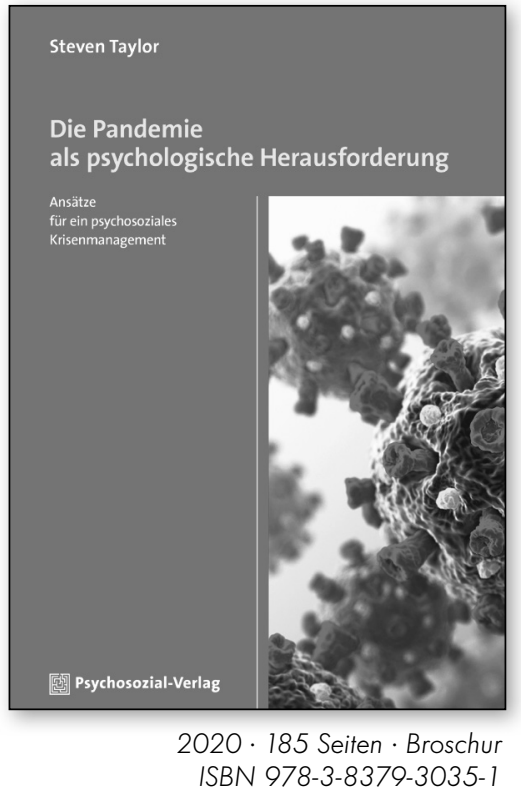

"Eine wertvolle Grundlage für politische Entscheidungsträger.»

Dean McKay

"Ein umfassender Überblick über die psychologischen Zusammenhänge und Folgen von Pandemien.» Bunmi O. Olatunji
Schon lange vor dem neuartigen Coronavirus wurden Szenarien für die Bekämpfung von Pandemien entworfen. Psychologischen Faktoren und emotionalen Belastungen wurde dabei bemerkenswert wenig Aufmerksamkeit zuteil. Mit der Zielsetzung, diese psychosoziale Dimension stärker zu beleuchten, erschien im Herbst 2019 die englischsprachige Originalausgabe dieses Buches nur wenige Wochen vor dem Ausbruch von COVID-19 im chinesischen Wuhan.

Auf der Grundlage der wissenschaftlichen Literatur zu früheren Pandemien untersucht Steven Taylor die psychologischen Folgen von Pandemien und ihrer Bekämpfung. Er verdeutlicht, dass die Psychologie bei der (Nicht-)Einhaltung von Abstandsregelungen und Hygieneempfehlungen sowie beim Umgang mit der pandemischen Bedrohung und den damit verbundenen Einschränkungen eine wichtige Rolle spielt. Anhand zahlreicher Fallberichte erörtert er die vielfältigen Reaktionen: weitverbreitete Ängste vor Ansteckung und wirtschaftlichem Ruin, Panikkäufe, Verschwörungstheorien, Rassismus, unangepasstes Verhalten sowie Abwehrreaktionen, aber auch die Zunahme von Altruismus. 


\section{Moralisieren im Alltag}

\section{Editorial}

Journal für Psychologie, 28(2), 3-11

https://doi.org/10.30820/0942-2285-2020-2-3

CC BY-NC-ND 3.0 DE

www.journal-fuer-psychologie.de

Als im März 2020 Deutschland zur Eindämmung der Corona-Pandemie in den sogenannten »Lockdown « ging, wurde der Einkaufswagen zur moralischen Arena: Wer zu viel Nudeln, Mehl oder Toilettenpapier im Wagen hatte, war strafenden Blicken, bisweilen auch verbalen Angriffen ausgesetzt. Etwas, das für gewöhnlich klammheimlich oder zumindest kommentarlos stattfindet - die Inspektion und moralische Bewertung der Einkäufe anderer -, wurde nun in einem Gefühl eingetretener oder erwarteter Knappheit offen betrieben. Der Handel reagierte mit einer zumindest vermeintlich klaren Ansage: Ausgewählte Güter durften fortan nur noch in haushaltsüblichen Mengen eingekauft werden. Die Corona-Pandemie hat in vielen Bereichen des öffentlichen Lebens moralische Gebote explizit gemacht, die vorher in abgeschwächter, bisweilen auch deutungsbedürftiger und dynamischer Form bestanden haben.

\section{Moralisieren im Alltag - Zwischen Moral und Moralismus}

In einer sich als plural und liberal verstehenden Gesellschaft ist der wertende Blick auf den Einkauf anderer trotz seiner Banalität möglicherweise ein treffendes Beispiel für Moralisierungen im Alltag. Auch wenn Menschen klare moralische Standpunkte vertreten und an ihren Maßstäben andere messen, so bleibt ein offenes Moralisieren dennoch häufig aus. Wenn es erfolgt, so ist oft gleichzeitig die moralische Frage und auf einer Metaebene die Legitimität des Moralisierens Gegenstand der Auseinandersetzung. Alltagssprachlich ist der Begriff des Moralisierens entsprechend negativ konnotiert (Möhring-Hesse 2013). Dass auf Moralisierungen verzichtet wird, ist dabei nicht immer nur eine respektvolle Praxis, die der Wahrung des friedlichen Zusammenlebens mit Andersdenkenden dient. Vielleicht ebenso wichtig ist der Einfluss von sozialen Ungleichheiten und Machtstrukturen auf den Raum des Sag- und öffentlich Bewertbaren. Gleichzeitig sind in unterschiedlichen Lebensbereichen auch explizite Artikulationen moralischer Vorstellungen und Standpunkte zu hören, die mit der bisweilen leise, mitunter auch lautstark vorgetragenen Forderung verbunden werden, diese anzuerkennen und ihnen praktisch Rechnung zu tragen. Die Alltagskommunikation ist durchtränkt 
von starken Werthaltungen, normativen Perspektiven und ethisch-moralischen Selbstund Weltverständnissen. Manchmal sind sie allgemein gehalten und richten sich an alle.

Erscheinungs- beziehungsweise Ausdrucksformen der Moral und Moralisierung im Alltag - als Kommunikation, Interaktion, Aushandlung, Selbstthematisierung (ob verbal, textuell, visuell, face-to-face oder virtuell) - können enger oder loser, impliziter oder expliziter an Werte und moralische Orientierungen rückgebunden sein und darüber hinaus unterschiedliche psychosoziale und soziokulturelle Funktionen erfüllen. Es deuten sich motivierende und emanzipatorische Potenziale der Moralisierung von Lebensformen und Handlungsweisen ebenso an wie repressive Wirkungen im Zeichen moralischer Macht.

Das vorliegende Heft widmet sich den nur latent gefühlten, für sich behaltenen oder hinter vorgehaltener Hand geäußerten Moralisierungen ebenso wie den Momenten, in denen sie offen zur Sprache kommen und teils mit Vehemenz vorgebracht werden. Die Frage nach der Bewertung und damit der Legitimität des Moralisierens spielt dabei in fast allen Beiträgen eine Rolle. Manche der in den unterschiedlichen empirischen Materialien zu Wort kommenden Akteur*innen moralisieren - und setzen sich selbstreflexiv mit ebendieser Praxis auseinander. Andere hingegen meiden das Risiko, etwa als Moralist*in abgestempelt zu werden, und behalten ihre Werthaltungen weitgehend für sich.

\section{Moralisieren im Alltag - eine Herausforderung für die Psychologie}

Die Psychologie hat eine lange Tradition der wissenschaftlichen Beschäftigung mit Moral, am bekanntesten sind die anthropologischen Entwürfe einer Psychologie der Moral im Kontext der genetischen Epistemologie (Piaget 1986 [1932]; Kohlberg 1996). Diese primär auf Kognitionen und die Entwicklung von Moral im Lebenslauf abhebenden $\mathrm{Zu}$ gänge spielen im vorliegenden Themenheft allerdings eine weniger prominente Rolle. Es sind vornehmlich handlungstheoretische Perspektiven, die praktische und affektive Aspekte der Moral und Moralisierung in den Blick nehmen und die unternommenen Untersuchungen in ihrer Bedeutung für die (nicht nur) psychologische Theoriebildung reflektieren. In der Analyse des Moralisierens ist die Psychologie letztendlich auf ihre Nachbardisziplinen angewiesen und positioniert sich als sozial-, kultur- und geisteswissenschaftlich orientierte Disziplin.

Die wissenschaftliche Beschäftigung mit Moral und Moralisieren im Alltag muss es nicht dabei belassen, den Blick nach außen zu richten. Forschen selbst kann als Alltag betrachtet werden. Der Blick nach innen macht schnell deutlich, dass sich zum Beispiel auch Implikationen für die Ausgestaltung empirischer Forschungsmethoden ergeben 
können, die sich aufgrund ihrer Alltagsnähe handlungspraktisch wohl nie gänzlich von Moralisierungen entkoppeln lassen. Letztendlich kann jedwedes Forschungshandeln aus dieser Perspektive reflektiert werden. Dabei kann es sich um solitäre (Selbst-)Reflexionen der forschenden Personen ebenso handeln wie um die Organisation des mehr oder weniger expliziten sowie formalisierten Umgangs mit moralischen Positionen von Forschenden beziehungsweise deren Projekten.

Die Psychologie hat es seit jeher auch mit moralisch umstrittenen Phänomenen zu tun. Hinsichtlich mancher Untersuchungsgegenstände spielen Moral und Moralisieren eine allenfalls marginale Rolle, bei anderen stehen sie mit im Zentrum. Dass sich die Disziplin nicht willentlich am Moralisieren beteiligen sollte, gilt vielleicht als weitgehender Konsens. Sich indes ganz herauszuhalten, wird wohl auch nicht immer möglich sein, gerade wenn sie in intensiveren Austausch mit der Gesellschaft tritt (Stichwort Third Mission). Für manche Forschungsprojekte ist von Beginn an abzusehen, dass ein moralisches Minenfeld betreten wird. In anderen stellt sich dies erst im Prozess heraus.

Dies trifft auch auf die Projekte zu, die den Beiträgen dieses Themenheftes zugrunde liegen. Durch Analyse und Theoretisieren begeben sich die Autor*innen in unmittelbare Nähe dieser Felder und treten gleichzeitig zurück und nehmen (ein-)ordnende Distanz ein.

\section{Zu den Beiträgen des Themenheftes}

Ein auch nur annähernd umfassender Ordnungsversuch für die theoretische Betrachtung und empirische Analyse von Moral und Moralisieren in der psychologischen Forschung kann im Rahmen eines mehr oder weniger kontingent entstandenen Themenheftes selbstredend nicht unternommen werden. Was es im besten Falle zu leisten vermag, ist, selektive Einblicke in unterschiedliche Zugänge zu eröffnen und zur weiteren Systematisierung einzuladen.

Für das vorliegende Themenheft war es uns ein Anliegen, eine Bandbreite an Beiträgen zu gewinnen, die sich dem Phänomen sowohl theoretisch als auch auf Grundlage bereichsbezogener empirischer Untersuchungen nähern. Uns erreichten viele Manuskripteinreichungen, von denen acht letztendlich als Beiträge ins Themenheft aufgenommen werden konnten und die - zwar zwangsläufig ausschnitthaft und nur einige wenige der möglichen theoretischen Perspektiven, methodologisch-methodischen Zugänge und empirischen Felder berücksichtigend - einen eindrücklichen Facettenreichtum bieten. Sie stammen von Autor*innen aus Deutschland, Österreich und der Schweiz, die sich mitunter erst in jüngerer Zeit dem Thema »Moralisieren « zugewandt haben beziehungsweise durch ihre Forschung mit diesem konfrontiert wurden, und auch von solchen, für die es schon viele Jahre einen Schwerpunkt darstellt. 
Die Beiträge verweisen auf unterschiedlichste Dimensionen der Moral und des Moralisierens im Alltag: etwa die Omnipräsenz von Moral und Moralisierung im Tun und Lassen, ihre affektiven Qualitäten, die Relevanz (sub-)kultureller Differenzen für moralische Positionierungen, die Situativität moralischer Kommunikation, die interaktive Hervorbringung moralischer Standpunkte, die historische und biografische Prozesshaftigkeit moralischer Perspektiven, die temporale Dimension des Moralisierens, die diversen beabsichtigten und nicht-intendierten psychosozialen Folgen und Nebenfolgen moralischer Kommunikation, deren strategische Nutzung, und nicht zuletzt das ihr innewohnende gesellschaftliche Transformationspotenzial. - Im Folgenden stellen wir sie kurz vor.

In dem Beitrag »Haltung bewahren! Ressentiment als verkörpertes, implizit-moralisches Urteil « von Markus Wrbouschek, Natalie Rodax, Katharina Hametner, Sara Paloni und Nora Ruck spielen implizite Werthaltungen eine zentrale Rolle, die sich bisweilen nur durch körperliches Unwohlsein äußern. Aufbauend auf einer kompakten Fallvignette entwickeln die Autor*innen eine phänomenologisch-sozialpsychologische Theorie der Psycho- und Soziogenese des Ressentiments. Im Zentrum ihrer Analyse steht die Verschiebung von Affekten und latenten Werthaltungen aus ihrem ursprünglichen, sozialen Kontext in eine zumindest äußerlich davon unabhängige Situation. Haben sich die so verschobenen Affekte zu einem Ressentiment entwickelt, ist nur noch schwer erfahr- und reflektierbar, was an der ursprünglichen Situation »nicht stimmte «. Um an den Ursprungsmoment eigenen Unwohlseins zurückzukehren, ist sowohl ein genaues Nachfühlen und Erinnern an die eigenen Affekte als auch eine Analyse des sozialen Kontexts und seiner Machtverhältnisse notwendig. Damit entwerfen die Autor*innen eine Theorie, die zu einer vielschichtigen Analyse von Ressentiments und Werthaltungen einlädt.

Mit impliziten Formen des Moralisierens setzt sich auch Ralph Sichler in seinem Beitrag »Strukturanalyse normativ-praktischer Rationalität: Theoretische und methodologische Reflexionen zur interpretativen Rekonstruktion moralischer Orientierung « auseinander, für den er einen handlungstheoretischen und kulturpsychologischen $\mathrm{Zu}-$ gang wählt. Bezugnehmend auf die philosophischen Arbeiten von Julian Nida-Rümelin argumentiert er, dass menschliches Handeln sowie Überzeugungen und emotionale Reaktionen immer auch einen moralischen Gehalt aufweisen. Er legt dar, dass es zur Aushandlung moralischer Orientierungen nicht immer eines explizit moralischen Diskurses bedarf. Vielmehr dient Alltagskommunikation dem Austausch »guter Gründe « für das eigene Handeln und das Tun und Lassen der Mitmenschen - auch wenn es bisweilen vordergründig nur darum geht, einen bestimmten Ist-Zustand treffend zu beschreiben. An dieser Stelle konvergieren die theoretischen Überlegungen von Ralph Sichler zum Moralisieren im Alltag mit methodologischen Grundannahmen interpretativer Sozialforschung. In beiden Ansätzen stehen der Wunsch und die Suche nach 
Begründung des Handelns im Zentrum. Diese Gründe beziehungsweise Begründungen werden allerdings weder immer kommunikativ angekündigt, noch während des Handlungsvollzugs oder ihm nachgelagert explizit gemacht. Vielmehr liegt die Aufgabe interpretativer Forschung in der Aufdeckung ebendieser Begründungsmuster in qualitativen Daten wie beispielsweise in Interviews und Gruppendiskussionen. Ralph Sichler verknüpft also philosophische Überlegungen zum Moralisieren mit konkreten empirischen Forschungsmethoden. Dies leitet gelungen zu den empirischen Beiträgen dieses Themenheftes über, die Moralisierungen im Alltag interpretativ analysieren.

In ihrem Beitrag »Moralisierungen in Gastfamilien für unbegleitete Geflüchtete: Differenzbearbeitung und Beziehungsgestaltung zwischen Gast und Familie « zeichnet Ines Gottschalk am Einzelfall nach, wie eine Gastmutter sich im Interview mit ihren eigenen moralischen Vorstellungen - und den Vorstellungen anderer - von geschlechtergerechter Arbeitsteilung im Haushalt auseinandersetzt. Dabei zeigt sich die Mehrdeutigkeit des Begriffs »Gastfamilie«, der unbegleitete Geflüchtete einerseits als Gast, andererseits als Familienmitglied konzeptualisiert. Mit diesen Rollen gehen unterschiedliche Vorstellungen und Ansprüche an die Integration in die Familie und die Aushandlung geteilter moralischer Orientierungen einher. Ines Gottschalk veranschaulicht an diesem Beispiel, dass Moralisierungen im Alltag in komplexe soziale Beziehungen eingebunden sind und wie Kompromisse eingegangen werden. Die Einzelfallanalyse macht deutlich, dass durch interkulturelle Begegnungen und Differenzerfahrungen auch eigene moralische Positionierungen in Bewegung geraten können.

Moralische (Re-)Positionierungen und Abgrenzungen zu anderen spielen auch eine zentrale Rolle in der Längsschnittstudie von Frank Zahradnik. Im Beitrag »Biografische Dynamiken moralischer Positionierungen in Prozessen sozialer Reintegration - Eine Längsschnittanalyse im Kontext von Drogenkonsum, Kriminalität und Arbeit « beschreibt er anhand von vier narrativen Interviews, die im Abstand von jeweils eineinhalb Jahren mit einem wegen Drogendelikten strafrechtlich verurteilten Mann geführt wurden, die dynamische biografische Entwicklung der Selbstpositionierungen. Besonders interessant ist, wie es dem Interviewten gelingt, sich positiv von anderen abzugrenzen, obwohl er aufgrund seines Drogenkonsums, der strafrechtlichen Verurteilung und der Inanspruchnahme verschiedener staatlicher Hilfesysteme mit negativen sozialen Kategorisierungsprozessen konfrontiert ist. Dabei spielen seine hohe Arbeitsmotivation und die Aufrechterhaltung von Mindestanforderungen einer »normalen « Lebensführung (zum Beispiel Verhinderung von Wohnungslosigkeit) für ihn eine zentrale Rolle. Die Längsschnittdaten ermöglichen es zu sehen, wie die Vergleiche mit und Abgrenzungen von anderen in Bewegung geraten, wenn sich die eigene Lebenssituation, zum Beispiel durch die Aufnahme einer regulären Arbeit, ändert. 
Geronnene und starke Formen moralischer Abgrenzung stehen im Zentrum des Beitrags $\gg>$ Egal was er auch ist, Hauptsache einer von uns< - Eine sozial- und kulturpsychologische Analyse zur Bedeutung von Moral im alevitisch-sunnitischen Verletzungsverhältnis « von Dilek $A$. Tepeli. Sie spürt Moralisierungen nach, die zunächst als von einer anderen Gruppe ausgehende moralische Be- und Abwertungen erscheinen, sich sodann jedoch als historisch gewordene und sich wechselseitig vollziehende Praxis erweisen, in der sich die Gruppen jeweils Moral absprechen. Auf Grundlage der Analyse einer Gruppendiskussion mit zwei in Deutschland lebenden jungen alevitischen Frauen wird gezeigt, wie das historische kollektive Verletzungsverhältnis zwischen Alevit*innen und Sunnit*innen selbst im postmigrantischen Kontext die moralisch abwertende Bezugnahme aufeinander prägt. Die moralische Kommunikation in dieser Intergruppenbeziehung wird als Aktualisierung eines seit Jahrzehnten, ja Jahrhunderten etablierten ein- und ausgrenzenden Sprechens mit- und vornehmlich übereinander kenntlich. Damit liegt der Fokus des Beitrags auf den negativen psychosozialen Funktionen (Auf- und Abwertung, In- und Exklusion) der moralischen beziehungsweise moralisierenden Selbst- und Fremdthematisierung als Fortschreibung des intergruppalen Verletzungsverhältnisses. Die Bedeutung der Antizipation, als alevitische Frau durch einen sunnitischen Ehemann zur Adressatin moralisierender und bisweilen missionierender Rede zu werden, wird am Beispiel einer von den Gesprächspartnerinnen imaginierten sunnitisch-alevitischen Intergruppenehe dargestellt. Der Beitrag verweist damit auf die temporale Dimension des Moralisierens, das mehr oder weniger explizite und komplexe Bezugnahmen auf Vergangenes, Gegenwärtiges und Zukünftiges beinhalten kann.

Die Situativität und gemeinschaftliche Hervorbringung moralischer beziehungsweise moralisierender Positionen betont der Beitrag »>DA wurd ich Auch ganz blöde angeguckt<. Das moralische Spannungsfeld zwischen gesellschaftlichem Anspruch und individueller Integrität in einer Selbsthilfegruppe zum Thema Übergewicht « von Sarah Hitzler. Sie schließt an die Konzeptualisierungen von Bergmann und Luckmann (2013a [1999], 2013b [1999]) an, indem sie Moral nicht in der Person verortet und als raum-zeitlich ungebundenes Phänomen essenzialisiert, sondern die Funktionen von Moralisierungen als Ausdruck von Moral in Interaktionen in den Blick nimmt. Auf Grundlage des Transkripts der Sitzung einer Selbsthilfegruppe für Personen mit starkem Übergewicht arbeitet sie mittels eines konversationsanalytischen Zugangs heraus, wie Moralisierungen narrativ-interaktiv hervorgebracht werden und dabei sowohl situativen kommunikativen und psychosozialen Zwecken dienen, als auch an geteilten sowie zurückgewiesenen gesellschaftlichen Normen orientiert sein können. Die Moralisierungen werden dabei an wechselnde Wertesysteme geknüpft. Wie die Analyse zeigt, ermöglicht die Vagheit der narrativen Darstellungen es den Gruppenmitgliedern, mitunter stark konfligierende Norm- und Wertvorstellungen 
sowie moralische Positionen zu verhandeln und gleichzeitig den Gruppenzusammenhang aufrechtzuerhalten.

Das gesellschaftliche Transformationspotenzial des Moralisierens und damit verbundene positiv konnotierte Zukunftsorientierungen betonen die beiden letzten Beiträge des Themenheftes, einmal aus empirischer, einmal aus theoretischer Perspektive. Auf Grundlage von (Paar-)Interviews mit Veganer*innen geht Rebecca Thrun in ihrem Beitrag »Jenseits des guten Geschmacks? Veganisierung im Alltag « der Frage nach, wie diese ihre Überzeugungen hinsichtlich eines ethisch-moralischen Veganismus als verallgemeinerungsfähige und -würdige Lebensform in Interaktionen im sozialen Umfeld einbringen. Die Analyse zeigt, dass die Kommunikation dabei mit unterschiedlichsten Facetten des Moralisierens durchzogen ist. So sehen sich Veganer*innen bisweilen gefordert, ihr auf Transformation (individuelle Veränderung als auch gesellschaftlicher Wandel hin zu einer veganen Lebensweise) abzielendes Handeln von abgewerteten beziehungsweise abgewehrten Formen der Überzeugungsarbeit, wie etwa Bekehrung, Missionierung, Provokation, Konfrontation, moralische Anklage, abzugrenzen sowie einer Positionierung als Dogmatiker*in und Moralist*in entgegenzuwirken. Auf rationaler Ebene entwickeln sie rhetorisch-argumentative und auf affektiver Ebene selbstregulative Strategien, mit denen die Hoffnung verbunden wird, eine wirkmächtigere Überzeugungsarbeit zu leisten. Diese relativiert zwar die Auffassung der moralischen Überlegenheit der eigenen Lebensform und ist von zumindest partieller kommunikativer Offenheit geprägt, jedoch nicht frei von Machtausübung und manipulatorisch-instrumentellem Zugriff auf sich und andere. Die Bezugnahmen auf moralische Überzeugungen einer veganen Lebensform erweisen sich als polyvalentes Unterfangen.

In ihrem Beitrag »Das klimaethische Selbst als Subjektivierungsform - Eine idealtypische Konstruktion « widmet sich Alexandria Krug aus einer von der angewandten Ethik inspirierten Perspektive der Thematisierung von Moralisierungstendenzen in Zeiten des Klimawandels als (auch) ethisch-moralische Herausforderung menschlichen Handelns. Besonders hervorgehoben werden dabei klimaethische (vor allem Klimagerechtigkeit) und verantwortungstheoretische Aspekte (insbesondere individuelle Verantwortung und Langzeitverantwortung). Die theoretische Auseinandersetzung und idealtypische Konstruktion behandelt nicht nur einen ethisch-moralischen, normativ aufgeladenen Gegenstand, sondern ist selbst normativ angelegt. Moralisierungstendenzen werden in diesem Beitrag vornehmlich als willkommene Impulse für als wünschenswert erachtete Veränderungsprozesse in den Blick genommen, wenn klimabezogene Moralansprüche zu ethisch abgewogenen Selbstansprüchen transformiert werden. Das klimaethische Selbst wird als handlungsfähiges Subjekt entworfen, das sich durch weitreichende Verantwortungsübernahme auszeichnet und (selbst-)reflexiv (klima-)ethische Perspektiven aneignet. Der Beitrag lädt als Heuristik zur empirischen 
Untersuchung eines solchen - vornehmlich auf Rationalität bauenden, historisch, (sub-)kulturell und gesellschaftlich kontingenten - Ideal-Entwurfs ein, um ihn etwa hinsichtlich divergierender klimarelevanter Handlungsfelder auszudifferenzieren und in seiner Akzentuierung von Vernunft und individueller Handlungsmacht zu befragen.

Wir hoffen, dass die hier versammelten Beiträge dazu anregen mögen, künftig sowohl in noch weiteren Feldern dem Moralisieren nachzuspüren, als auch die bereichsübergreifenden Perspektivierungen und Theoretisierungen systematisierend auszubauen.

Ihnen, liebe Leser*innen, wünschen wir eine spannende Lektüre von Beiträgen zu einem Thema, welches 2020 zu einem noch aktuelleren wurde, als wir es uns bei der ersten Planung des Themenheftes im Frühjahr 2019 hätten je vorstellen können.

Paul S. Ruppel \& Anna Sieben

\section{Dank}

Wir möchten uns ganz herzlich bei den Gutachter*innen für ibre kritischen Anmerkungen, hilfreichen Hinweise und konstruktiven Stellungnabmen bedanken. Obne diese weitgehend unsichtbar bleibende und überaus verdienstvolle Arbeit wären Themenhefte wie das vorliegende bekanntlich nicht denkbar. Dass die Gutachter*innen trotz mitunter sehr widriger Corona-bedingter Umstände durch ihre Tätigkeit zum Erscheinen dieses Themenheftes beigetragen haben, wissen wir sehr zu schätzen. Unser Dank gilt ebenso dem Psychosozial-Verlag und hier namentlich Christian Flierl für die Organisation des Lektorats sowie allen Herausgeber*innen des Journalsfür Psychologie, die unsere Themenheftidee vom Moment ibrer Äußerung an unterstützt haben.

\section{Literatur}

Bergmann, Jörg und Thomas Luckmann, Hrsg. 2013a [1999]. Kommunikative Konstruktion von Moral. Band 1: Struktur und Dynamik der Formen moralischer Kommunikation. Mannheim: Verlag für Gesprächsforschung.

Bergmann, Jörg und Thomas Luckmann, Hrsg. 2013b [1999]. Kommunikative Konstruktion von Moral. Band 2: Von der Moral zu den Moralen. Mannheim: Verlag für Gesprächsforschung.

Kohlberg, Lawrence. 1996. Die Psychologie der Moralentwicklung. Frankfurt a. M.: Suhrkamp.

Möhring-Hesse, Matthias. 2013. »Moralisieren sierung. Perspektiven kritischer Sozialer Arbeit, hrsg. von Ruth Großmaß und Roland Anhorn, 151-165. Wiesbaden: Springer VS.

Piaget, Jean. 1986 [franz. Orig. 1932]. Das moralische Urteil beim Kinde. München: DTV. 


\section{Die HerausgeberInnen}

Paul Sebastian Ruppel, Dipl.-Psych., wissenschaftlicher Mitarbeiter am Lehrstuhl für Sozialtheorie und Sozialpsychologie an der Fakultät für Sozialwissenschaft, Ruhr-Universität Bochum, und freier Mitarbeiter im Institut für Qualitative Forschung in der Internationalen Akademie Berlin. Forschungs- und Interessensschwerpunkte: Qualitative Forschung; Kulturpsychologie; Identitätsforschung; Klimawandel und Mobilität. Paul Sebastian Ruppel hat zu sozial- und kulturwissenschaftlichen Themen und zur Methodologie und Methodik qualitativer Forschung veröffentlicht. Seit 2006 ist er Mitherausgeber des Journals für Psychologie und seit 2016 Associate Editor bei Forum Qualitative Sozialforschung / Forum: Qualitative Social Research.

Kontakt: Paul Sebastian Ruppel, Ruhr-Universität Bochum, Fakultät für Sozialwissenschaft, Lehrstuhl für Sozialtheorie und Sozialpsychologie, Gebäude GD 1/219, Universitätsstr. 150, D-44801 Bochum; E-Mail: Paul-Sebastian.Ruppel@rub.de

Anna Sieben, Dr., Dipl.-Psych., ist Leiterin der Abteilung "Sozialpsychologie der Fußgängerdynamiken« am IAS-7 des Forschungszentrum Jülich sowie wissenschaftliche Mitarbeiterin am Lehrstuhl für Sozialtheorie und Sozialpsychologie der Ruhr-Universität Bochum. Ihre Forschungsschwerpunkte sind: Menschenmengen und Fußgänger*innen, Elternschaft, Psychologisierungen des Alltags, qualitative Methoden, Kulturpsychologie, kulturvergleichende Forschung, feministische und queere Psychologien.

Kontakt: Dr. Anna Sieben, Forschungszentrum Jülich, Civil Safety Research (IAS-7), Wilhelm-Johnen-Str., D-52425 Jülich; E-Mail: an.sieben@fz-juelich.de 